\title{
On Relationship between Pediatric Shi Ji and Fever
}

\author{
Xiangyu Hu*, Lina Hu \\ Pingdingshang City Hospital of Traditional Chinese Medicine, Pingdingshan, China \\ Email: pds-hxy025@163.com
}

Received 24 June 2015; accepted 4 September 2015; published 7 September 2015

Copyright (C) 2015 by authors and Scientific Research Publishing Inc.

This work is licensed under the Creative Commons Attribution International License (CC BY).

http://creativecommons.org/licenses/by/4.0/

cc) (i) Open Access

\begin{abstract}
Based on the clinical effect of the treatment on 546 Pediatric Shi Ji fever cases, the thesis tries to explore the effectiveness of Traditional Chinese Medicine(TCM) treatment on Pediatric Shi Ji and the relationship between Pediatric Shi Ji and fever. The methodology applied is a retrospective analysis on the clinical curative effect of TCM treatment on Shi Ji fever cases in our hospital from January 2008 to December 2012. And the results show that a total effective rate of $96.3 \%$ could be guaranteed through either oral Chinese Medicinal Herbs, Chinese Medicine Enema, Massage Therapy, or navel administration with TCM. The thesis holds that Pediatric Shi Ji may cause fever, which could be cured simply by applying TCM treatment (promoting digestion to eliminate stagnation) with less or no use of antibiotics.
\end{abstract}

\section{Keywords}

TCM, Pediatric Shi Ji, Fever, Retrospective Analysis

\section{Introduction}

Similar to digestive function disorder in western medicine, Shi Ji (Indigestion with Food Retention) is a common kind of pediatric symptom, which can result in poor appetite, dyspepsia, diarrhea, constipation, even fever and sometimes multiple organ function damage such as respiratory system, cardiovascular system, nervous system, etc. For the last six years, as high as 546 fever cases caused by Pediatric Shi Ji have been diagnosed in our Hospital as follows:

\section{Materials and Methods}

\subsection{Source}

566 pediatric cases from our hospital in-patient department (233 cases) and outpatient department (312 cases)

${ }^{*}$ Corresponding author. 
from January 2008 to December 2012; Within which, 73 cases aged from 1 to 6 months, 186 cases aged from 7 to 36 months, 148 cases aged from 4 to 7 years, and 49 cases from 8 to 14 years old; Temperature: about 368 cases between $37.5^{\circ} \mathrm{C}$ and $38.5^{\circ} \mathrm{C}, 246$ cases between $38.5^{\circ} \mathrm{C}$ and $38.9^{\circ} \mathrm{C}, 50$ cases above $40^{\circ} \mathrm{C}$.

\subsection{Methods}

Ruling out other causes of fever and giving the corresponding treatment in accordance with the classification of indigestion with food retention. Using treatments like traditional Chinese herbal medicine, preparation, chiropractics, enema and massage therapy with less or no use of antibiotics and antipyretic drugs. Based on the observatory findings of the heat fading conditions and the possible complications, the thesis tries to make further summary, analysis, induction and consolidation.

\subsection{Traditional Chinese Medicine (TCM) Zhu San}

For two or more symptoms like nausea, vomiting, abdominal distension, distending pain, thick white coating, it belongs to the food retention in the stomach (basic type) and can be treated with Chiropractic Therapy, sanjia, shenling and baikou; For symptoms like vermilion, red tongue, irritability, feverish sensation in the palms and soles, yellow tongue, it belongs to the heat type of children food retention and can be treated with two more reagent like detoxification and calculus bovis; for two or more symptoms like hypodynamia, Emaciation, yellow or white dry coating, and thin sloppy stool, it belongs to the spleen injury type of children food retention and also needs combine with astringent prescription, etc.

\subsection{Other Treatments}

1) Natrii powder $3 \mathrm{~g}$, pepper 0.5 g, grinded into powder and mixed white vinegar into paste, daily dressing change. Used for heavier infantile indigestion with food retention.

2) Radix et Rhizoma Rhei 30 g, mirabilite 20 g, grinded into powder and then mixed white vinegar into paste, daily dressing change. Applied for symptoms like abdominal distension, abdominal pain and constipation caused by indigestion.

3) Vinasse $100 \mathrm{~g}$, fried hot, put into two bags, alternatively fomenting abdomen. 1 time per day, 2 to 3 hours each time. Used for spleen deficiency syndrome caused by indigestion.

4) Massage Therapy: Push and knead Banmen point 100 times, Qing dachang 100 times, Zhong wan 100 times, Fu yinyang 50 times, and massage abdominal for 2 minutes, Push and knead Zong sanli 100 times, Qi jiegu 100 times, push Spine 10 times and chiropractic 3 - 5 times. For children with inner Ji of milk food, nip Si feng 10 times, Na dujia 3 - 5 times, or combined with needling Si feng. For children with spleen deficiency syndrome caused by indigestion, Push and knead Pitu 100 times, Yun Shui Ru Tu 100 times.

5) Chinese Medicine Enema, Endothelium corneum, medicated leaven, malt, parched hawthorn fruit, radish seed, dried tangerine or orange peel, poria cocos, pinellia, Fried hovenia dulcis, Radix Aucklandiae, etc. for Children with heavy internal heating, mixed with Coptis chinensis, plaster stone, Capejasmine, Forsythia, etc. 2 times a day, and 100 - $200 \mathrm{ml}$ for retention enema.

\subsection{Standard for Treatment Effect}

Cured: 24 - 48 hours after medication, both temperature and diet return to normal, other symptoms disappear; Significant effective: 24 - 48 hours after medication, temperature returns to normal, diet almost back to normal, most of the main symptoms disappear. Effective: 73 - 96 hours after medication, temperature is significantly lowered, main symptoms are eased. Invalid: either physical signs or symptoms are not changed.

\section{Results}

For the 566 pediatric cases, 354 cases were cured in 2 or 3 days by taking TCM Zhu san; 123 cases that have difficulty in taking drugs were treated with Chinese medicine enema and were cured in 3 days; the rest 49 cases were cured in 3 to 4 days through not only enema, but also the mixed use of massage therapy and navel administration with Chinese traditional medicine; 20 cases with other symptoms or poor effects were transferred to other treatments. In sum, we have up to $96.3 \%$ total effective rate. 


\section{Discussions}

The Word Shi Ji (Indigestion with food retention), appeared for the first time in book Confucians' Duties to Parents [1]. Shortly after, another book, Symptom Disease Cause Pulse Manifestation Therapeutic Methods, specifically spent several chapters discussing the following symptoms which are, Shiji Kesou or Dyspeptic coughs (Cough caused by Indigestion) [2]; Shiji Xiexie or Dyspeptic diarrhea (diarrhea caused by indigestion); Shiji Futong or Dyspeptic abdominalgia (Abdominal pain caused by indigestion) [2] and so on. Then much attention has been paid to symptoms caused by indigestion. Shen Jinao in Qing Dynasty took a further look on the disease: "most pediatric diseases are caused by indigestion of the milk food, or by the six pathogenic factors (wind, cold, summer heat, humidity, dryness and fire) combined with dyspepsia. So Shi Ji is specially listed out and is different from Jipi (literally, Indigestion addiction)" [3]. Shi Ji is resulted from improper feeding. The stagnated food stops in stomach and intestine and leads to spleen dysfunction, which in the end causes spleen-stomach or in other words, digestive system disease. Clinically, it is characterized with no appetite, abdominal distention, eructation with fetid odor, and sour stool or constipation. Shi Ji is also called stagnation, which is similar to indigestion or digestive disorder in Western medicine. The symptom may occur throughout the year and has a higher incidence in summer and autumn due to the summer damp-heat traps the spleen qi. Children of all age groups can be onset but with more frequency in infants, and the disease is often complicated with cold, diarrhea and malnutrition. Infants who have spleen-stomach weakness, innate deficiency or experience artificial feeding are easily attacked by such disease repeatedly. A minority of children who suffer long-time dyspepsia and persistent loss of treatment will have their spleen and stomach function badly damaged, which in turn hinders the absorption of nutrition and the growth of body, and reduces into Gan (malnutrition). That is the reason why our forefathers expressed this way: "Ji is the mother of Gan, if there is no Ji, Gan will be non-existent." Causes and Manifestations of Children's Miscellaneous Diseases is the book that originally recorded the disease by using the term Dyspepsia Syndrome and Hyperhagia [4]. After that Huo You Xin Shu applied Ji syndrome to name the disease while Ying Tong Bai Wen used Ji Zhi [5]. Another book Essentials of the Care of Infants Cold-Heat Syndrome of Shi Ji) clearly pointed out that Pediatric Shi Ji is resulted from "long-term indigestion of the milk food because of the deficiency cold in spleen and stomach" [6].

As the living standards continue to improve, more and more types of food come onto people's table. In order to supply their children with nutrition, mothers and fathers blindly give their children nutritional supplements. Such high intensity has had serious impact on children's stomach and spleen function. The infantile period is a growing stage for children, during that period many of their body's functions are not in shape, especially the digestive system function is relatively weak, the secretion of digestive fluid is too little and therefore the gastrointestinal absorption ability is very poor. And what's more, children can not control themselves like adults, if parents feed them a bit carelessly; it is likely to cause digestive function disorder. When the milk food comes into the stomach, it can not be properly digested and absorbed, which will damage both stomach and spleen function, and will cause indigestion with food retention. After a period of time this will be transformed into heat, if combined with indigestion, will produce internal heat. If continued, it is bound to lead to fever accompanied by symptoms such as abdominal pain, bloating, belching, sour stool, constipation, and even symptoms like dysphoria, poor sleep, sweating for some children. Based on previous experiences, we can say the fever caused by infantile Shi Ji is not characterized by high temperature but low, and with the feeling of thirst.

Through recent years' observation, we have found it is not uncommon for some of such high fever cases lasting for a long period of time [7] [8]. The treatment of this fever should focus on promoting digestion to eliminate stagnation. Fever will naturally disappear if the stagnation is eliminated. In addition, other treatments like massage, chiropractic therapy could also be used to cure the fever effectively. Western medicine believes that for children, their digestive function is not yet perfect, bacteria planted in their intestinal and antibodies are relatively fewer than normal; indigestion can cause gastrointestinal dysfunction, lower immunity, gastric acid secretion decrease, which in turn causes food indigestion; and besides, enteric pathogenic bacteria breed quickly and are absorbed into the bloodstream through the intestinal mucosa, which may cause fever, multiple organ damage and many complications, even have life-threatening consequence to the children.

According to Chinese medicine, fever caused by Shi Ji, is divided into two categories: exogenous fever and fever of internal injury. The former is initiated by the struggle between vital qi of human body and exogenous pathogenic factors perceived by human body. The latter is related to factors like diet, tiredness or injury of seven emotions namely, joy, anger, worry, fear, love, hate and desire. These factors may result in viscera dysfunction, 
Qi-blood imbalance, and excess of either yin or yang, which in turn bring about fever. For children, the circulation of vital energy is not sufficient; the viscera are very delicate, their liver and intestine functions are good, yet the spleen is aesthetically susceptible. And besides, children's feeling of hunger is not quite obvious, they can't tell if it is enough for food and drinks. If given unsuitable or coarse food, they are easily hurt. Pediatric pathogenesis has the following characteristics: they get sick easily; the symptom spreads and changes rapidly; food retention transforms into internal fever easily and can lead to the coexistence of fever and indigestion. Clinically, such cases are more common and the chief complaint is fever. Some doctors often treat the fever with antibiotics, which not only fails to cure the fever, but causes gastrointestinal symptoms, even allergic reactions after the use of antibiotics and antipyretic. In the past, due to the limitation of living condition, there was a shortage of nutrients, even if the function of spleen and stomach is very good, malnutrition still could not be prevented because of the shortage. Now the living standard is obviously improved and the majority of families have only one child, which make the parents overindulge their children. What's more, Media mislead the public to take more nutritional Foods; some parents even force their children to eat, which causes children have strong negative mentality. As time passes it develops into indigestion with food retention, seriously malnutritional stagnation and even all kinds of disease.

Through the discussion of the connection between fever and pediatric indigestion with food retention, the paper tries to explore the theoretical relationship of both Western medicine and Chinese medicine in the area. And furthermore, In order to enhance children's physical quality, reduce the occurrence of disease, and promote the healthy growth of children, the paper tries to give tips on how to feed children scientifically, as well as how to treat the fever by Chinese medicine with less or no use of antibiotics.

\section{References}

[1] Zhang, H.C., et al. (1984) Revised Confucians’ Duties to Their Parents. Henan Scientific and Technical Publishers.

[2] Qin, J.M. (1990) Symptom Disease Cause Pulse Manifestation Therapeutic Methods. Shanghai Scientific and Technical Publishers, Shanghai.

[3] Shen, J.N. (1980) You Ke Shi Mi. Shanghai Scientific and Technical Publishers, Shanghai.

[4] Chao, Y.F. (2008) Treatise on Causes and Manifestations of Various Diseases. Huaxia Press, Beijing.

[5] Sun, H.S. (2005) Golden Mirror of Medicine. Youke Xinfayaojue Baihuajie. People’s Medical Publishing House, Beijing.

[6] Chen, F.Z. (2006) You You Ji Cheng. People’s Medical Publishing House, Beijing.

[7] Wang, H.T. (2010) The Self-Made Soup Reach of the Original Cold Solution Heat Treatment 36 Cases of Children with Food Product Efficacy. Journal of Traditional Chinese Medicine, 03, 41-542.

[8] Liang, J.J. (2004) The Heat-Clearing and Eliminate Stagnation Method Reach of the Original Cold Solution Heat Treatment 30 Cases of Children with Food Product Efficacy. Journal of Emergency in Traditional Chinese Medicine, 10, 640 . 